This item was submitted to Loughborough's Research Repository by the author.

Items in Figshare are protected by copyright, with all rights reserved, unless otherwise indicated.

\title{
Coupling the six flux absorption-scattering model to the Henyey-Greenstein scattering phase function: Evaluation and optimization of radiation absorption in solar heterogeneous photoreactors
}

\section{PLEASE CITE THE PUBLISHED VERSION}

http://dx.doi.org/10.1016/j.cej.2016.04.127

\section{PUBLISHER}

(C) Elsevier

\section{VERSION}

AM (Accepted Manuscript)

\section{PUBLISHER STATEMENT}

This work is made available according to the conditions of the Creative Commons Attribution-NonCommercialNoDerivatives 4.0 International (CC BY-NC-ND 4.0) licence. Full details of this licence are available at: https://creativecommons.org/licenses/by-nc-nd/4.0/

\section{LICENCE}

CC BY-NC-ND 4.0

\section{REPOSITORY RECORD}

Acosta-Herazo, Raul, Jesus Monterroza-Romero, Miguel Angel Mueses, Fiderman Machuca-Martinez, and Gianluca Li Puma. 2016. "Coupling the Six Flux Absorption-scattering Model to the Henyey-greenstein Scattering Phase Function: Evaluation and Optimization of Radiation Absorption in Solar Heterogeneous Photoreactors". figshare. https://hdl.handle.net/2134/22281. 


\section{COUPLING THE SIX FLUX ABSORPTION-SCATTERING MODEL TO THE HENYEY-GREENSTEIN SCATTERING PHASE FUNCTION: EVALUATION AND OPTIMIZATION OF RADIATION ABSORPTION IN SOLAR HETEROGENEOUS PHOTOREACTORS}

Raúl Acosta-Herazo ${ }^{\mathrm{a}}$, Jesús Monterroza-Romero ${ }^{\mathrm{a}}$, Miguel Ángel Mueses ${ }^{\mathrm{a}, *}$, Fiderman Machuca-Martínez ${ }^{\mathrm{b}}$ and Gianluca Li Puma ${ }^{\mathrm{c}}$

${ }^{a}$ Photocatalysis and Solar Photoreactors Engineering, Department of Chemical Engineering, Universidad de Cartagena, A.A. 1382-Postal 195 Cartagena, Colombia

${ }^{\mathrm{b}}$ GAOX Research Group, School of Chemical Engineering, Universidad del Valle, A.A. 25360 Cali, Colombia

${ }^{\mathrm{c}}$ Environmental Nanocatalysis \& Photoreaction Engineering, Department of Chemical Engineering, Loughborough University, Loughborough LE11 3TU, United Kingdom

Corresponding author:

Professor Miguel Ángel Mueses, Ph.D.

Email:mmueses@unicartagena.edu.co

Telephone:+57-5-6752040 Ext 225 


\section{Abstract}

Robust and practical models describing the radiation field in heterogeneous photocatalytic systems, used in emerging environmental, photochemical and renewable energy applications, are fundamental for the further development of these technologies. The sixflux radiation absorption-scattering model (SFM) has shown to be particularly suitable for the modeling of the radiation field in solar pilot-plant photoreactors. In this study, the SFM was coupled to the Henyey-Greenstein (HG) scattering phase function in order to assemble the model with a more accurate description of the scattering phenomenon provided by this phase function. This new version of SFM, named as SFM-HG, was developed through fitting the Local Volumetric Rate of Photon Absorption (LVRPA) determined in a flat photoreactor to the "pseudo-experimental” LVRPA calculated by a Monte Carlo (MC) approach, which included the HG expression. As a result, simple mathematical correlations describing the SFM-HG scattering probabilities as function of the HG scattering parameter were determined. The SFM-HG was validated through a comparison with the MC model predictions of the Total Rate of Photon Absorption (TRPA) in the slab photoreactor. A RMSE\% of approximately 5\% demonstrated satisfactory agreement between the models. The SFM-HG was further applied to evaluate the impact of selected scattering phase functions on the absorption of radiation in solar photoreactors, operated with commercial $\mathrm{TiO}_{2}$ photocatalyst. The results have established that, the apparent optical thickness, $\tau_{\text {app }}$ or

$\tau_{\text {app,max }}$ for tubes, a parameter derived from the SFM approach, is the most appropriate parameter for the design and optimization of photocatalytic reactors, since such parameter is insensitive to scattering albedos and phase functions. CPC, tubular and flat-plate 
photoreactors operated with $\mathrm{TiO}_{2} \mathrm{P} 25$ should be designed with $\tau_{\text {app,max }}=12, \tau_{\text {app,max }}=7$ and $\tau_{\text {app }}=4.5$ respectively.

Keywords: $\mathrm{TiO}_{2}$-Photocatalysis, Titanium Dioxide, Local Volumetric Rate of Photon Absorption, Monte Carlo simulation, Radiative Transfer Equation.

\section{Abbreviations:}

DOM, Discrete Ordinate Method

HG, Henyey-Greenstein

LVRPA, Local Volumetric Rate of Photon Absorption

MC, Monte Carlo

SFM, Six Flux Scattering-Absorption Model

SFM-HG, Six Flux Scattering-Absorption Model coupled to Henyey-Greenstein phase function

RTE, Radiative Transfer Equation

TRPA, Total Rate of Photon Absorption

\section{Introduction}

As an emerging environmental technology, heterogeneous photocatalysis has received increasing attention in recent years. Its promising applications in air and water remediation [1], clean fuels production [2], green products (e.g. self-cleaning surface [3]) and selective 
synthesis of organic molecules [4] demonstrates great interest in this technology. The underlying basis of every photocatalytic reaction mechanism is the photoactivation of the semiconductor photocatalyst by absorption of photons with energy higher or equal than the catalyst band-gap. The consequent generation of electron-hole pairs produces a chain of reactions that drive simultaneous oxidation and reduction (redox) reactions.

The evaluation of the radiation field and of the spatial distribution of radiation absorption in a photoreactor system, commonly described by the local volumetric rate of photon absorption (LVRPA), is therefore a crucial aspect in the development of efficient photocatalytic processes [5].

The LVRPA is the photon equivalent to the concentration of reactant species and is always considered in the description of the kinetics of photochemical reactions and in the optimization of the performance of photoreactors. For instance, several methodologies have been proposed for the determination of the optimum catalyst load or reactor thickness which maximize the absorption of radiation [6,7].

The estimation of the LVRPA has been a defiant task within the heterogeneous photocatalysis community, as result of the complex nature of the absorption and scattering radiation phenomenon, which in the most rigorous case is modeled by the Radiative Transfer Equation (RTE). The RTE for a medium that does not emit radiation is [8]:

$$
\frac{d I_{\lambda}(s, \Omega)}{d S}=-\kappa_{\lambda} I_{\lambda}(s, \Omega)-\sigma_{\lambda} I_{\lambda}(s, \Omega)+\frac{\sigma_{\lambda}}{4 \pi} \int_{\Omega=4 \pi} p\left(\Omega^{\prime} \rightarrow \Omega\right) I_{\lambda}\left(s, \Omega^{\prime}\right) d \Omega^{\prime}
$$

where $I_{\lambda}$ is the photon irradiance at $\lambda$ wavelength, $s$ is a spatial coordinate, $\Omega$ is the directional solid angle, $\kappa_{\lambda}$ is the absorption coefficient and $\sigma_{\lambda}$ the scattering coefficient. The 
term $p\left(\Omega^{\prime} \rightarrow \Omega\right)$ is the scattering phase function representing the probability of a photon to be redirected by scattering from the direction $\Omega^{\prime}$, in surroundings of the position $s$, into the direction $\Omega$ [8]. A trivial solution for the RTE cannot be achieved and a numerical method is always necessary.

Although the precise modeling of the LVRPA and a better understanding of the radiationphotocatalyst-operational conditions nexus still remains a challenge, several approaches have been proposed to solve the RTE. The most rigorous methods are described by the Discrete Ordinate Method (DOM) [5,9] commonly included in CFD packages, and by the Monte Carlo (MC) stochastic method which yields an accurate representation of the radiation field [10,11]. Despite the modeling power of the previous methods, these are often mathematically and computationally demanding when applied at the solar dimension scale, which is invariably characterized by atmospheric fluctuations of the boundary conditions (i.e., photon flux), in turn increasing the complexity of the models. Hence these methods are generally limited to modeling systems with constant-flux artificial radiation sources (e.g., UV lamps) [12].

In contrast, the Six Flux Absorption-Scattering Model (SFM) has been proposed as a practical and simplified approach for solving the RTE. It is based on geometric optics but retains the key aspects of radiation field modeling characteristic of a rigorous approach. The SFM algebraic nature allows short computational times and low mathematical complexity, even when applied to complex geometries at solar scale as the tubular and the CPC photoreactor [13].

Brucato et al. [14] proposed the SFM for the calculation of the LVRPA in heterogeneous photoreactors under the hypothesis that scattering of photons occurs only along the six 
Cartesian directions. These directions were differentiated as forward, backward and sideward (orthogonal) and were assigned with scattering probabilities $p_{f}, p_{b}$ and $p_{s}$ as represented in Fig. 1. Their values depend on the phase function describing the photon scattering phenomena and have been estimated for the case of a diffuse reflectance (DR) phase function as $p_{f}=0.11, p_{b}=0.71$ and $p_{s}=0.045$ [14]. For isotropic scattering, SFM probabilities should all be equal to $p_{f}=p_{b}=p_{s}=1 / 6$ [15].

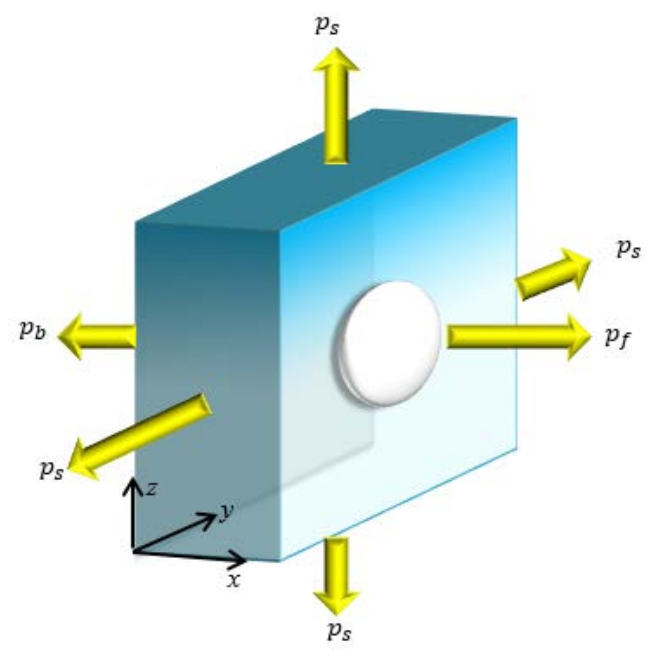

Fig. 1. Scattering directions considered by the SFM in a differential volume. The liquid is in blue, the scattering particle in white and the scattered light in yellow.

Albeit remains unclear which scattering phase function is the most adequate to model the radiation field in heterogeneous photocatalytic reactors, the determining role played by different formulations of these on the radiation field calculations has been shown [16], which makes its determination a significant step, as relevant as the prior knowledge of the absorption and scattering coefficients of the photocatalyst [17]. Table 1 lists the most common scattering phase functions used in photocatalysis and some of the most relevant studies in which were adopted. 
The DR phase function was originally considered in early studies for the estimation of the LVRPA in photoreactors. This theoretical phase function was borrowed from the radiation transport theory, since the optical characteristics (i.e. particle size and wavelength of photons absorbed) of titanium powders approximate the behavior of a diffuse reflective particle [18]. The expression for the DR phase function, with $\varphi$ as the exit angle of the scattered photon is [8]:

$$
p(\varphi)=\frac{3}{8 \pi}(\sin \varphi-\varphi \cos \varphi)
$$

However, subsequent studies suggested the isotropic phase function as more appropriate to model the scattering behavior of commercial titanium dioxide particles $[19,20]$. Since the pioneering work of Satuf et al. [21], the implementation of the Henyey-Greenstein (HG) phase function has been consolidating in literature, as an improvement to other expressions. The HG phase function is defined as follows [22]:

$$
p(\varphi)=\frac{1}{2} \frac{1-g^{2}}{\left(1+g^{2}-2 g \cos \varphi\right)^{3 / 2}}
$$

Where $-1 \leq g \leq 1$ is a scattering parameter indicating the anisotropy of the medium called asymmetry factor, negatives values correspond to backward scattering and positives values to forward scattering, isotropic scattering corresponds to $g=0$ [21]. The main contribution of HG function is that it removes the assumption about the scattering behavior of the particles implicitly made when a theoretical phase function is implemented, since it allows a previous experimental characterization of scattering thanks to its adjustable scattering 
parameter. A graphical representation of DR, isotropic and HG phase functions is reported in Figure SD1 of the Supplementary Data.

Notwithstanding the advantages of using the SFM for the modeling of the radiation field in solar photoreactors, previous studies have only been reported with the DR and/or the isotropic phase function, although the proven superiority offered by HG function. The SFM can indeed be formulated with any type of phase function, therefore, the aim of this study was to couple the SFM to the HG phase function combining the practicality of the SFM approach to the improvement in the description of the scattering phenomenon offered by the HG function. This new model is here referred to as "SFM-HG", in order to differentiate it from the SFM incorporating the diffuse reflectance phase function "SFM-DR" presented in earlier studies. The SFM-HG was fitted, using an optimization algorithm, to the "pseudoexperimental” data of LVRPA in a slab photocatalytic reactor with suspended photocatalytic particles obtained from a MC approach, which also considered the HG expression. The application of SFM-HG is outlined in this study through its implementation in the radiation field modeling of solar heterogeneous photoreactors.

Table 1 . Common scattering phase functions used in heterogeneous photocatalysis.

\begin{tabular}{llll}
\hline Phase & Applications & Contribution & Ref. \\
function & & & \\
\hline Diffuse & Determination of optical & Pioneer experimental method for \\
reflectance & properties by & optical properties. These were [18] \\
(DR) & spectrophotometry and & reported for $\mathrm{TiO}_{2}$ & \\
\end{tabular}


DOM.

Modeling photocatalytic Methods for kinetics, degradation of water optimization and scale-up of contaminants by SFM. annular tubular and CPC

photoreactors

Isotropic Modeling photocatalytic Approach for kinetics

(ISO) degradation of water independent of the reactor [15]

contaminants. $\quad$ conditions.

Henyey- Determination of optical HG phase function is proposed as

Greenstein properties by an improvement. Optical

(HG) spectrophotometry and properties reported for $\mathrm{TiO}_{2}$ with $[21,25]$

DOM. $\quad$ a tendency to forward scattering $g$

$>0.4$.

Modeling photocatalytic Kinetics and scale-up methods for degradation of organic annular photoreactors

compounds by DOM and

MC method.

Radiation field and Phase functions found crucial on evaluation of scattering for radiation field. HG parameter $\mathrm{TiO}_{2}$ suspensions in annular $0.6<g<0.8$ is reported as suitable $[16,30]$ photoreactor by $\mathrm{MC}$ method for $\mathrm{TiO}_{2}$ Optical properties by Experimental and numerical goniometry and CFD method for optical properties. The 
HG found as better for scattering

characterization than the DR. A

preferential forward scattering is

reported for $\mathrm{TiO}_{2}, \mathrm{~g}>0.5$.

\subsection{Slab geometry photoreactor setup}

A slab photoreactor geometry was considered for the fitting of SFM to the HG phase function. This geometry is commonly used in the formulation of radiation transport models, such as the SFM [14] or RTE [21,31]. It provides a one-dimensional approach of the phenomenon and a convenient handling of the resulting data. Furthermore, results obtained from the slab can be conveniently adapted to more complex geometries. For instance, the SFM was formulated for this geometry [14] and was extended to annular [32], tubular and CPC photoreactors [6]. The photoreactor considered in this work is as that described in Brucato et al. [14]: an infinitely high and wide slab solid-liquid photoreactor of thickness $L$, uniformly irradiated with photons arriving perpendicularly to the front wall, with an incident photon flux of $I_{0}$. As a result, the radiation field varies along the x-coordinate only (Fig. 2). 


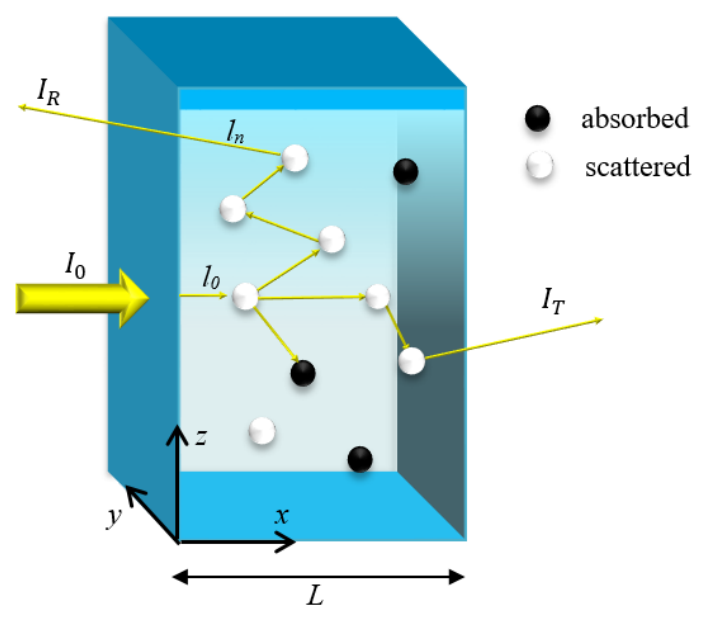

Fig. 2. Schematic diagram of the slab photoreactor with absorbing and scattering particles suspended in the inside. $I_{0}, I_{R}$ and $I_{T}$ are the incident, reflected and transmitted intensity of light, $L$ is the reactor length and $l$ the free path of photons.

\section{Mathematical methods}

\subsection{Monte Carlo radiation field model}

The Monte Carlo method allows the solution of complex radiation transport problems without making significant simplifications [33]. In fact, the intrinsic random nature of absorption and scattering of light makes it an ideal system to be modeled with MC. The MC model in the present work follows the methodology described in Pasquali et al. [34] and Brucato et al. [14]. The approach consists in establishing a statistically representative sample of photons and recording the history of each photon, from the moment it enters the system until its extinction (by absorption or scattering outside the system) including every interaction along the path. In order to make decisions about photons fates and trajectories, random numbers $(R)$ uniformly distributed in the interval $[0,1]$ were employed. The slab 
reactor computational domain was gridded in volume cells of thickness $d x$ to perform the simulations. The model was outlined according to the following steps:

i) Since photons irradiates the photoreactor normal to the front wall, each photon is emitted in a direction $\theta_{i}=0$ and assigned with the position $x=0$.

ii) The mean free path-length $l$, where the photon is neither absorbed nor scattered, is related to the extinction coefficient of the suspension $\beta$ according to the equation [12]:

$$
l=-\frac{1}{\beta} \ln R
$$

iii) The new position of the photon is calculated by geometry:

$$
x_{i}=x_{i-1}+l \cos \theta_{i}
$$

Where $i$ and $i-1$ refers to the actual and previous iteration of the algorithm. If $x$ $>L$ or $x<0$, the photon is deemed to be outside the geometry of the slab and it is considered lost. Then, a new photon is considered.

iv) Once the free path length is traveled by the photon, an event is considered to occur. Whether the photon is absorbed or scattered is determined by generating a random number $R$. The photon is absorbed and its location (cell) is recorded if $R$ is larger or equal to the scattering albedo $\omega$. If $R<\omega$ the photon is scattered and a new random direction is determined. $\omega$ which is determined by the ratio of the scattering and extinction coefficients of the photocatalyst suspension represents the probability of scattering [35]. 
v) If the photon is scattered, the new scattering direction is determined by the azimuth (longitude) and the zenith (latitude) angles. The azimuth is a variable uniformly distributed in the interval $[0,2 \pi]$, thus it can be sampled with the formula [36]:

$$
\alpha_{i+1}=2 \pi R
$$

The zenith angle $\varphi$ is provided by the HG phase function. In order to achieve a convenient form for the sampling of Eq. (3), this is rearranged as:

$$
p(\cos \varphi)=\frac{1}{2} \frac{1-g^{2}}{\left(1+g^{2}-2 g \cos \varphi\right)^{3 / 2}}
$$

Here the sampling variable is the cosine of the angle, thus the zenith is calculated as follows [22]:

$$
\cos \varphi_{i+1}= \begin{cases}\frac{1}{2 g}\left[1+g^{2}-\left(\frac{1-g^{2}}{1-g+2 g R}\right)^{2}\right], & \text { if } g \neq 0 \\ 2 R-1 & , \text { if } g=0\end{cases}
$$

The scattering direction is specified by the scattering angle $\theta$ relative to the $x$ coordinate, its corresponding cosine is defined by spherical trigonometry [37]:

$$
\cos \theta_{i+1}=\cos \varphi_{i+1} \cos \theta_{i}+\sin \varphi_{i+1} \cos \alpha_{i+1} \sin \theta_{i}
$$

Once the new direction is obtained, the procedure is reiterated from step ii.

The LVRPA is computed as a normalized magnitude under the hypothesis of complete radiation absorption within the reactor volume, simply by counting the number of photon 
absorbed in each volume cell divided by the incident radiation (represented by the total number of photons entering the reactor) and the reactor volume:

$$
\frac{L V R P A}{I_{0} / L}=\frac{N_{p h o t, \text { cell }} / d x}{N_{\text {phot, } \max } / L}
$$

Where $N_{\text {phot, cell }}$ and $N_{\text {phot, max }}$ are the photons absorbed in the $i^{\text {th }}$ cell and total number of photons in the simulation, respectively. The incident photon flux was set as $10^{6}$ photons, since it provided invariant results and short computational times. The flow chart for the MC model is reported in Figure SD2 of Supplementary Data.

\subsection{SFM-HG model}

\subsubsection{SFM structure}

The LVRPA divided by the incident radiation flux at a point $x$ in the photoreactor calculated with SFM for a plane geometry is [14]:

$$
\frac{L V R P A}{I_{0}}=\frac{1}{\lambda_{\omega_{\text {corr }}} \omega_{\text {corr }}(1-\gamma)}\left[\omega_{\text {corr }}-1+\sqrt{1-\omega_{\text {corr }}^{2}} e^{\frac{-x}{\lambda_{\text {corr }}}}+\gamma\left(\omega_{\text {corr }}-1-\sqrt{1-\omega_{\text {corr }}^{2}} e^{\frac{x}{\lambda_{\text {corr }}}}\right)\right]
$$

Where $a, b, \omega_{\text {corr }}$ and $\gamma$ are SFM parameters defined as follows:

$$
\begin{gathered}
a=1-\omega p_{f}-\frac{4 \omega^{2} p_{s}^{2}}{1-\omega p_{f}-\omega p_{b}-2 \omega p_{s}} \\
b=\omega p_{b}+\frac{4 \omega^{2} p_{s}^{2}}{1-\omega p_{f}-\omega p_{b}-2 \omega p_{s}} \\
\omega_{\text {corr }}=\frac{b}{a}
\end{gathered}
$$




$$
\begin{gathered}
\lambda_{\omega_{\text {corr }}}=\frac{1}{a \beta \sqrt{1-\omega_{\text {corr }}^{2}}} \\
\gamma=\frac{1-\sqrt{1-\omega_{c o r r}^{2}}}{1+\sqrt{1-\omega_{c o r r}^{2}}} e^{-2 \tau_{\text {app }}}
\end{gathered}
$$

Where the apparent optical thickness $\tau_{\text {app }}$ is:

$$
\tau_{\text {app }}=a \tau \sqrt{1-\omega_{\text {corr }}^{2}}
$$

The above SFM parameters are computed exclusively from the photocatalyst scattering albedo, reactor optical thickness and the scattering phase function. The scattering albedo is defined as:

$$
\omega=\frac{\sigma}{\beta}=\frac{\sigma}{\sigma+\kappa}
$$

where $\beta, \kappa$ and $\sigma$ are the photocatalyst specific extinction, absorption and scattering coefficients. The optical thickness is:

$$
\tau=L \beta C_{c a t}
$$

with $L$ and $C_{c a t}$ as the reactor length and catalyst load, respectively. The scattering phase function is specified in the SFM by the scattering probabilities: forward " $p_{f}$ ", backward " $p_{b}$ " and sideward " $p_{s}$ ". They must satisfy the SFM main principle, from their definition of probabilities [14]:

$$
p_{f}+p_{b}+4 p_{s}=1
$$




\subsubsection{SFM-HG structure}

In contrast to the DR or isotropic phase functions which indicate only a particular scattering mode and to which correspond unique values of $p_{f}, p_{b}$ and $p_{\mathrm{s}}$, the HG expression includes a range of scattering modes by means of its asymmetry factor $g$ which can vary in the interval $[-1,1]$. Therefore, the inclusion of this new phase function in the SFM should involve a way for the calculation of the SFM-HG scattering probabilities from the knowledge of the HG scattering parameter. This can be accomplished if it is postulated that a mathematical relation between the HG asymmetry factor and SFM-HG scattering parameters exists.

By considering SFM-HG as a function exclusively of the scattering probabilities, since scattering albedo and optical thickness are usually known, Eq. (11) could be written as:

$$
\frac{\text { LVRPA }}{I_{0}}=f_{S F M-H G}\left(p_{f}, p_{b}, p_{s}\right)
$$

with the SFM-HG scattering probabilities expressed as function of the HG parameter $g$, as follows:

$$
\begin{aligned}
& p_{f}=f_{1}(g) \\
& p_{b}=f_{2}(g)
\end{aligned}
$$

It should be noted that only two of the scattering probabilities are independent variables. $p_{f}$ and $p_{b}$ are chosen as independent because the anisotropy of scattering is referred as “forward” or "backward”, thus $p_{s}$ always can be obtained from Eq. (20). 


\subsubsection{Optimization procedure}

In order to determine $f_{1}$ and $f_{2}$, it is necessary to find the SFM-HG scattering probabilities for each value of $g$. On the basis of Eqs. (21-23), this is carried out by obtaining the LVRPA in the photoreactor for different values of the HG scattering factor along the interval $[-1,1]$ through the MC model and fitting the SFM-HG to each simulation. The information from the MC model is treated as "pseudo-experimental" in analogy to the classical fitting method in science and engineering in which experimental data are gathered to fit models. Models supported on MC simulations are usually used as gold standard for the evaluation of other numerical or analytical models [38].

The search for the best values of SFM-HG scattering probabilities that match each MC simulation is carried out by an optimization procedure. It starts with an initial guess for $p_{f}$, $p_{b}$ and $p_{s}$ and follows an optimization criterion until the required convergence is reached. In this study, it is proposed to minimize the error function given by the sum of the squared errors of the LVRPA calculated by both models:

$$
f_{\text {error }}\left(p_{f}, p_{b}, p_{s}\right)=\sum\left(\overline{L V R P A_{M C}}-\overline{L V R P A_{S F M-H G}}\right)^{2}
$$

subject to the limiting conditions:

$$
\begin{aligned}
& 0<p_{b}, p_{f}, p_{s}<1 \\
& p_{f}+p_{b}+4 p_{s}=1
\end{aligned}
$$

where $\overline{L V R P A_{M C}}$ denotes the normalized LVRPA profile along the slab photoreactor determined from the MC model for a given scattering albedo, optical thickness and HG asymmetry factor, and $\overline{L V R P A_{S F M-H G}}$ is the normalized LVRPA profile evaluated with the 
SFM-HG (Eq. (11) divided by reactor length $L$ ) for the same scattering albedo and optical thickness.

Eqs. (25) and (26) are constraints regarding the fundamental principles of the SFM and they must be satisfied. The system was solved using the Matlab optimization toolbox "fmincon" which determines the local minimum of a specified function subject to linear inequalities by an interior point algorithm.

The value of the product of the specific extinction coefficient and catalyst load (the volumetric extinction coefficient with units $\mathrm{m}^{-1}$ ) was assumed to solve the MC model and SFM-HG, and the reactor length was easily obtained from Eq. (19), in order to match the desired value for the optical thickness. Fig. 3 sketches the optimization procedure described. The purpose of using normalized LVRPA and dimensionless parameters is to make the results valid for any photocatalyst, reactor size or irradiance conditions. It has been shown that photoreactors with identical optical thicknesses and scattering albedos perform similarly regarding the absorption of radiation [6]. 


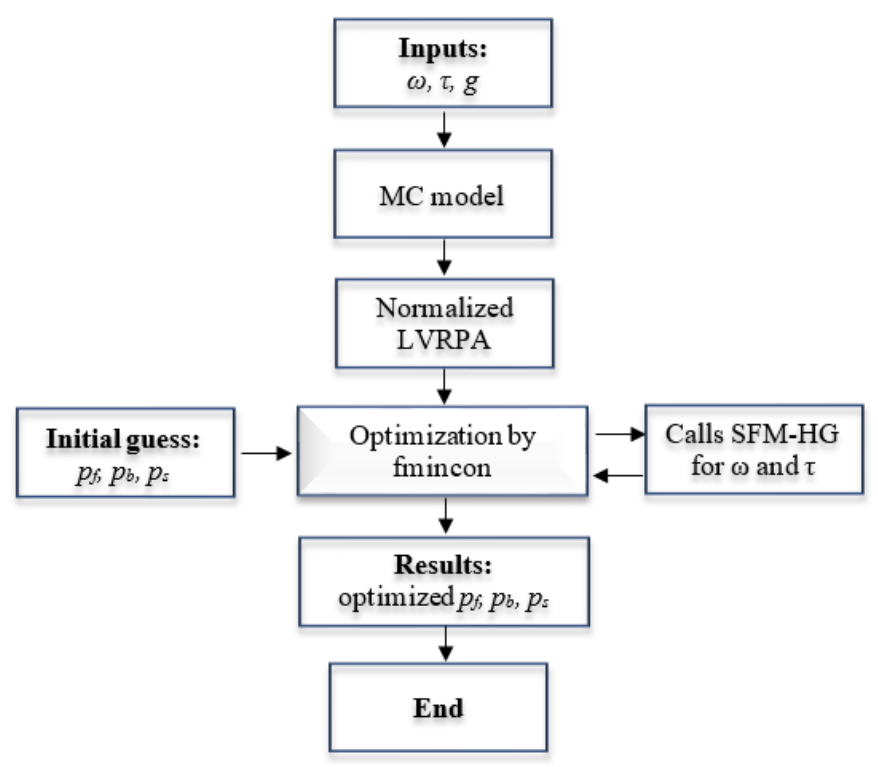

Fig. 3. Schematic representation of the optimization procedure used to fit the SFM-HG for each value of $g$.

\section{Results and discussion}

\subsection{Determination of correlations for SFM-HG scattering parameters}

Fig. 4 shows the trend of the SFM-HG scattering probabilities relative to the HG asymmetry factor determined from the optimization procedure. A tendency to follow a linear dependence is evident for both the backward and forward scattering probabilities, with deviations at the extreme values of $g$ approaching -1 and 1 . Therefore, a linear fitting of the simulation data is proposed as a mathematical representation for the $f_{1}$ and $f_{2}$ functions in Eqs. (22) and (23) which take the form:

$$
p_{f}=0.4467 g+0.5193
$$




$$
p_{b}=\left\{\begin{array}{rr}
-0.4692 g+0.3805 & -1 \leq g \leq 0.8 \\
0 & g>0.8
\end{array}\right.
$$

with correlation coefficients of $\mathrm{R}^{2}=0.99$ for $p_{b}$ and $\mathrm{R}^{2}=0.988$ for $p_{f}$.

Furthermore, from Eq. (20), the side scattering probability equals:

$$
p_{s}=\frac{1}{4}\left(1-p_{f}-p_{b}\right)
$$

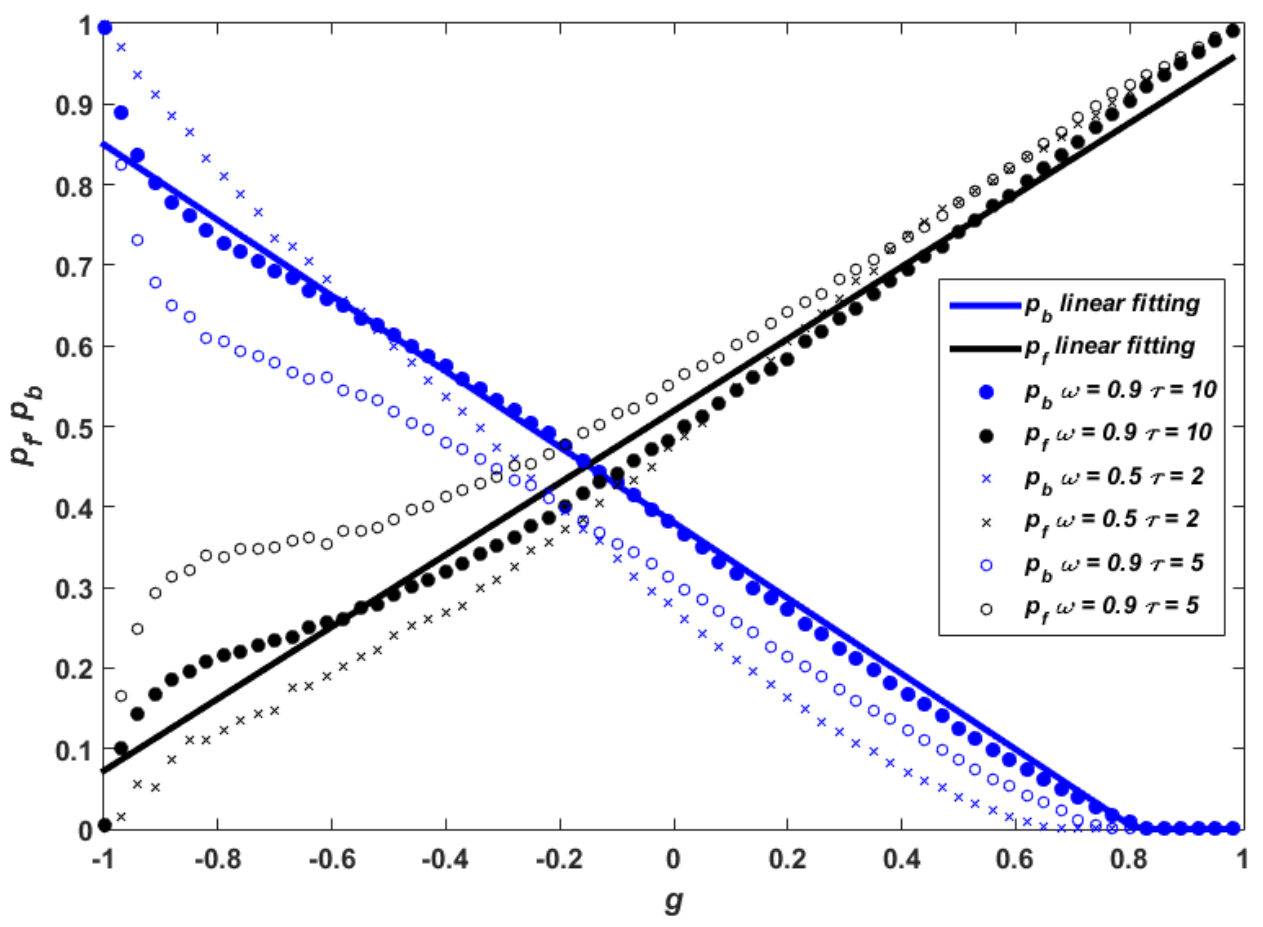

Fig. 4. Fitted SFM-HG scattering probabilities as function of the HG asymmetry factor and linear fitting of the data.

Eq. 27 and 28 corresponded to the linear fitting for scattering albedo $\omega=0.9$ and optical thickness $\tau=10$ in Fig. 4. These optical properties were selected to ensure that SFM-HG is 
able to match the limit conditions of the radiation field for a slab geometry photoreactor, following Brucato et al. approach in SFM formulation [14]. Besides, $\omega=0.9$ can be considered as an arbitrary average value for the scattering albedo of the photocatalyst titanium dioxide reported in literature $[7,24]$. Different combinations of these optical parameters $(0.1 \leq \omega \leq 0.95$ and $2 \leq \tau \leq 12)$ were tested and yielded close results (Fig. 4$)$ in

the confidence interval of $-0.8 \leq g \leq 0.8$. The observed deviations from the linear fitting in Fig. 4 are expected to be compensated when smaller scattering albedos and optical thicknesses are considered in radiation field simulations.

Eqs. (27-29) coupled with Eq. (11) conform the novel SFM-HG. As shown in Fig. 4, the contrasting slopes for $p_{f}$ and $p_{b}$ are in agreement with the HG phase function model, which predicts an increase of forward scattering with an increase of the asymmetry factor $g$, and vice versa.

\subsection{Validation of SFM-HG}

The validation of radiation-transport photoreactor models against the MC model is an approach widely adopted in literature [14,41]. The gathering of experimental data to determine the spatial-dependent magnitudes of the LVRPA can be very complex, since the absorption and scattering of radiation by the system is often disturbed by the measuring device [39]. Accordingly, in this study the SFM-HG that was calibrated at optical thickness $\tau=10$ and scattering albedo $\omega=0.9$, was validated with data from MC simulations of the radiation field collected at different values of these optical parameters.

Fig. 5 compares the transversal profiles of the normalized LVRPA in the slab photoreactor calculated with the MC and SFM-HG models for different combinations of scattering albedos, optical thicknesses and asymmetry factors. It shows the profound effect of the 
asymmetry factor $g$ on the radiation field in the slab, as scattering switches from predominantly back-scattering to predominantly forward scattering. The discrepancies observed in Fig 5a up to $x / L=0.1$ are due to a local maxima predicted by the MC model and not shown by the SFM-HG. These have also been observed by Brucato et al. [14] for the DR phase function (black line in Fig. 5a) and also predicted from DOM simulations [19]. This maxima found at such high values of scattering albedo and optical thickness in a slab photoreactor, results probably because under these conditions since the reactor is optically “thick". A perfect agreement between SFM/SFM-HG and MC or DOM simulation should not be expected, since the former considers that scattering only occurs in six-directions, in order to gain practicality over the more complex MC and DOM models. By comparing Fig. 5a and Fig. 5b, the accuracy of the SFM-HG improves at smaller scattering albedos, a feature also observed by Brucato et al. [14] on the SFM. 

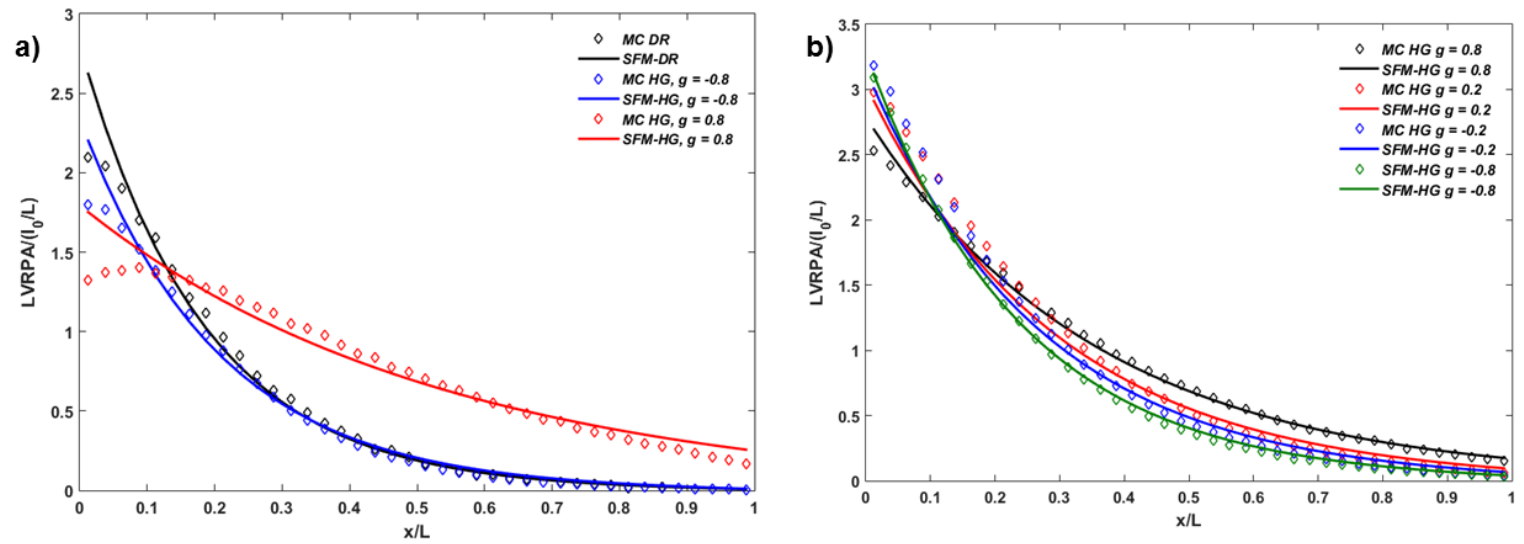

Fig. 5. Normalized LVRPA across the slab photoreactor as function of the scattering phase function determined with the MC model, the SFM-HG and the SFM-DR. a) $\omega=0.9$ and $\tau=$ 10. b) $\omega=0.5$ and $\tau=5$ MC-DR and SFM-DR refers to simulations performed with the Diffuse Reflectance phase function.

The SFM-HG was further validated via the estimation of the Total Rate of Photon Absorption (TRPA) which is the overall rate of radiation absorbed in the entire reactor volume. The TRPA has shown useful in the analysis of photocatalytic systems [16,23]. For a flat photoreactor the TRPA normalized by the incident photon flux is:

$$
\frac{\text { TRPA }}{I_{0}}=\int_{0}^{L} \frac{L V R P A}{I_{0}} d x
$$

The normalized TRPA profiles, as illustrated in Fig. 6a provides a clear graphical representation of the effect of scattering albedo and optical thickness on the TRPA. It shows an increase with optical thickness up to reaching a plateau at $\tau$ values progressively increasing with higher values of $g$. In contrast, the results in Fig. 6b shows that the TRPA plateau is reached at the same $\tau_{\text {app }}=4.5$, regardless of the value of the HG asymmetry factor, which should be taken as reference for design and operation of flat photoreactors. A 
close value of $\tau_{\text {app }}$ has also been reported in literature for the annular geometry [35]. The results shown in Fig. 6 reports good agreement between SFM-HG and MC model, independently of the optical thickness $\tau$ and the HG asymmetry factor $g$. Because of the integral nature of the TRPA, discrepancies in this variable between MC model and SFMHG can be considered as integral errors, since the models estimate directly the LVRPA and not the TRPA.
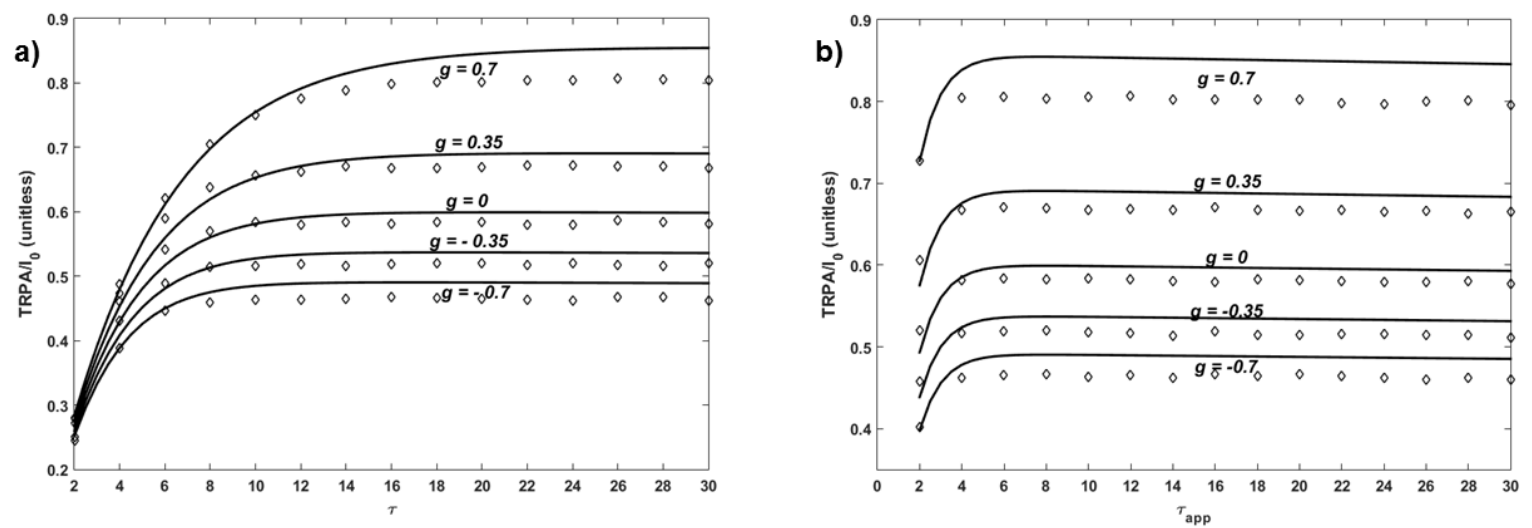

Fig. 6. Normalized TRPA for a slab photoreactor as function of the HG asymmetry factor $g$ calculated with the MC model (diamonds) and SFM-HG (lines). Optical properties: $\omega=$ 0.9 .

The accuracy of the SFM-HG calculations against the MC calculations for varying scattering albedo, optical thickness and HG asymmetry factor was numerically evaluated using the Root Mean Square Percentage Error (RMSE\%) of the TRPA profiles:

$$
R M S E \%=\sqrt{\frac{1}{N} \sum_{i=1}^{N}\left(\frac{\overline{T R P A_{M C, i}}-\overline{T R P A_{S F M-H G, i}}}{\overline{T R P A_{M C, i}}}\right)^{2}} * 100
$$


where $\overline{T R P A_{M C, i}}$ and $\overline{T R P A_{S F M-H G, i}}$ are the normalized TRPA for the $i^{\text {th }}$ optical thickness calculated with the MC and SFM-HG models respectively, and $N=15$ is the total number of optical thicknesses sampling values considered (Fig. 6). Table 2 shows the RMSE\% of the TRPA profiles for different scattering albedos and HG asymmetry factors. Low values (around 5\%) for RMSE\% were observed.

Table 2. RMSE\% of TRPA profiles estimated with the SFM-HG relative to MC model

\begin{tabular}{cccc}
\hline HG scattering factor & RMSE\% & RMSE\% & RMSE\% \\
$(g)$ & $\omega=0.3$ & $\omega=0.6$ & $\omega=0.9$ \\
\hline-0.7 & 0.96 & 1.6 & 4.62 \\
-0.35 & 0.95 & 2.45 & 3.38 \\
0 (Isotropic) & 1.74 & 3.74 & 3.3 \\
0.35 & 1.68 & 3.61 & 3.18 \\
0.7 & 0.49 & 1.18 & 5.39 \\
\hline
\end{tabular}

\subsection{Isotropic scattering}

The case of isotropic scattering $(g=0)$ offers an ideal point of reference to evaluate the accuracy of the SFM-HG model proposed in this study. The scattering probabilities estimated with the SFM-HG with Eqs. 27-29 results in $p_{f}=0.519, p_{b}=0.381$ and $p_{s}=$ 0.025, which differ from the theoretical values of the SFM for isotropic scattering (SFMISO), $p_{f}=p_{b}=p_{s}=1 / 6=0.167$ (i.e. scattering probabilities must be equal [35]). These discrepancies between SFM-ISO and SFM-HG are attributed to the divergence in the 
directional approach for scattering adopted in MC simulations and SFM. Despite both models are 3-dimensional, the MC model considers scattering to occur in N-directions while SFM in 6-directions only. Thus, the theoretical $p_{b}$ and $p_{f}$ for isotropic scattering of the SFM hardly match with those obtained from fitting the SFM-HG to the MC model.

Figs. 7 and 8 show the normalized profiles of the LVRPA and TRPA for the slab calculated from the MC model and the SFM-ISO and SFM-HG model prediction for isotropic scattering. The SFM-ISO appears to overestimate the radiation absorption in the photoreactor by approximately $10 \%$ at such high value of the scattering albedo $(\omega=0.9)$, with the error diminishing at smaller albedos [14]. It is further observed that both models do not predict the maxima observed at very small $x / L$ values by the $M C$ model, which gives a rigorous representation of the radiation field, although SFM-ISO fails by a larger margin. The more accurate representation of the radiation field by the SFM-HG model originates from fitting it to the MC model in the first instance, to determine the dependence of the scattering probabilities from the asymmetry factor $g$ (Eqs. (27-29)). A more accurate fit of the MC model results with the SFM has been shown for a diffusively reflecting phase

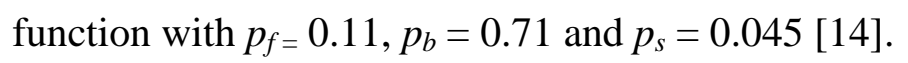




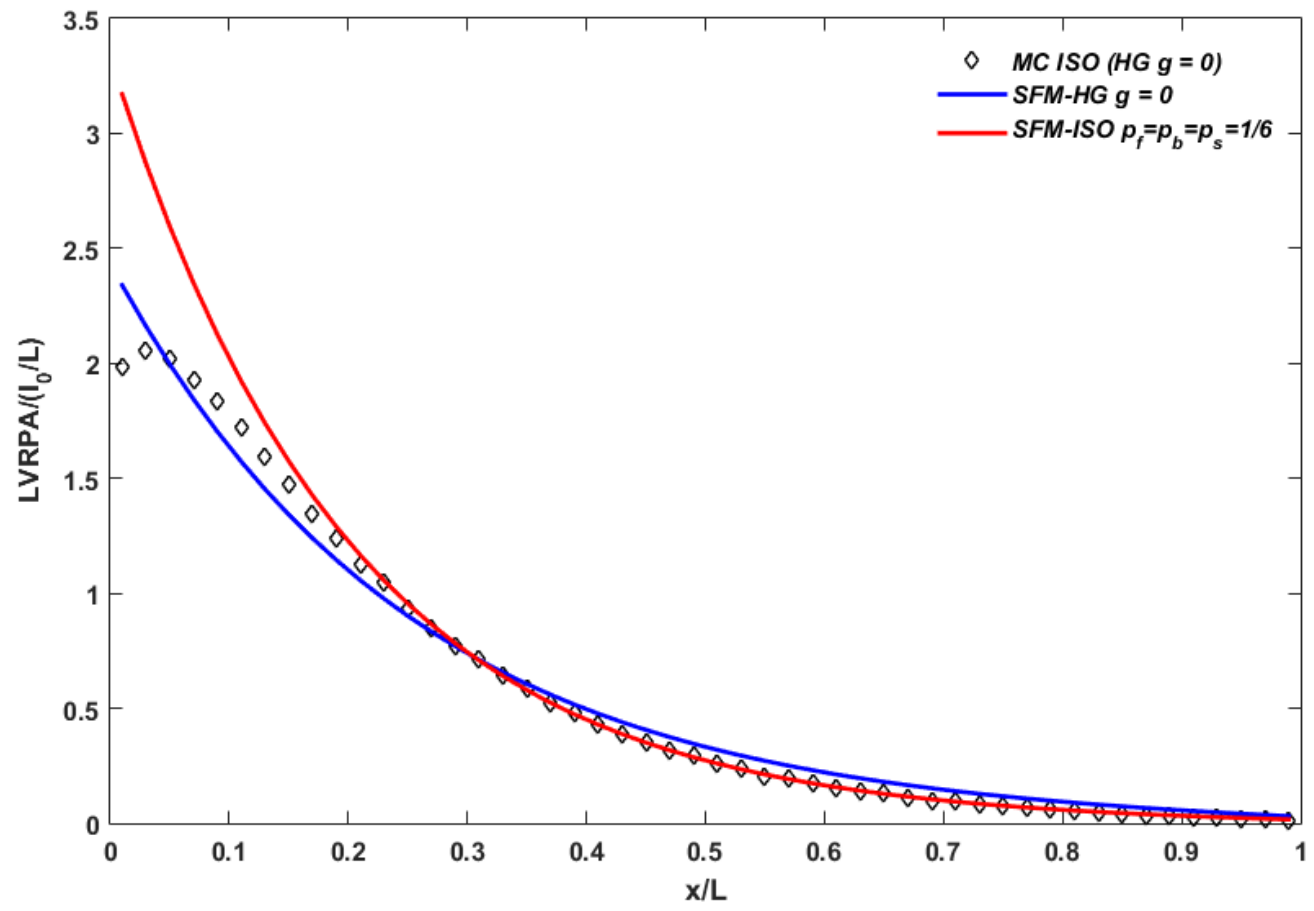

Fig. 7. Normalized LVPRA in the slab photoreactor. MC model (diamonds) and SFMISO/SFM-HG (lines). Optical properties $\omega=0.9$ and $\tau=10$. 


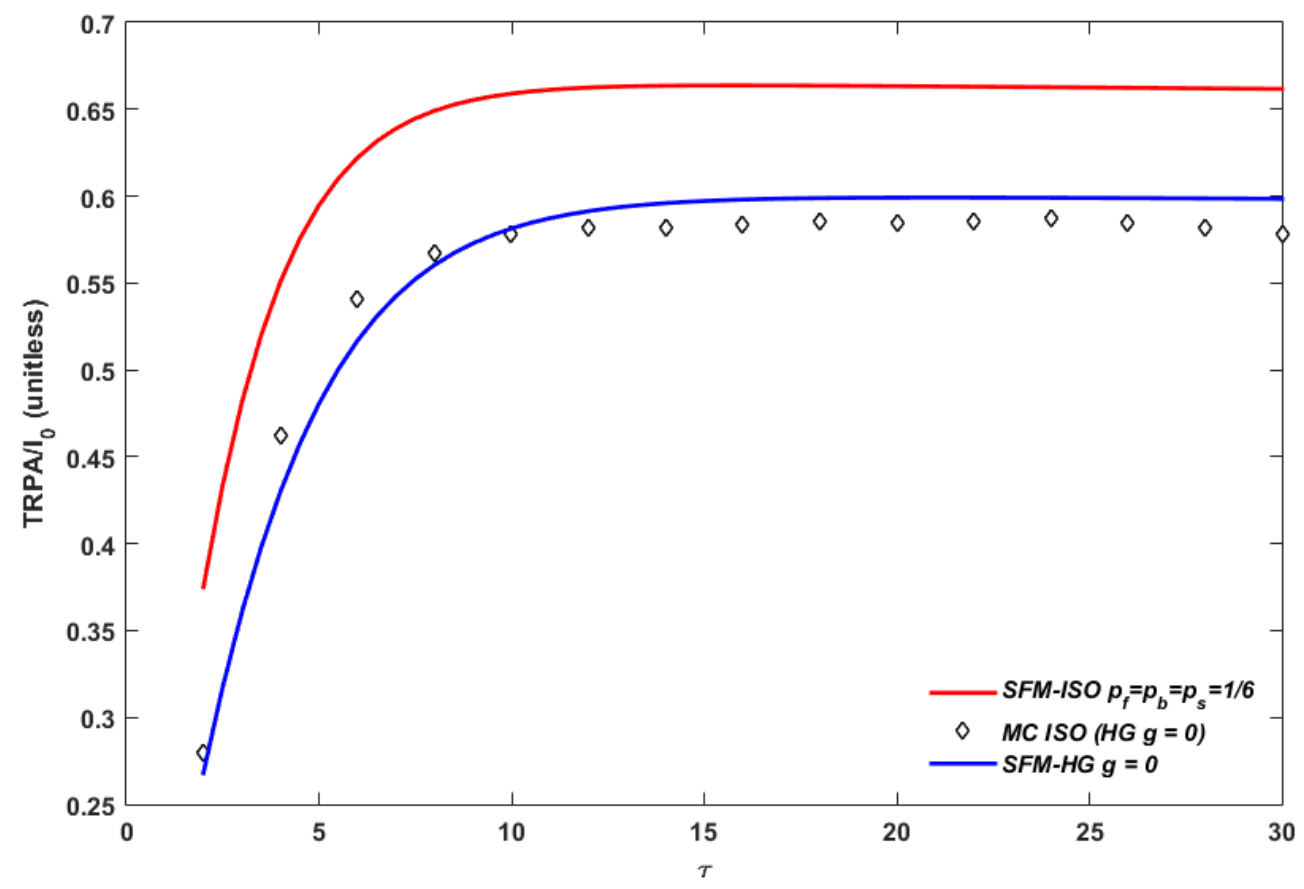

Fig. 8. Normalized TRPA for a slab photoreactor. MC model (diamonds) and SFMISO/SFM-HG (lines). Optical properties $\omega=0.9$.

\subsection{Application of SFM-HG to solar heterogeneous photocatalytic reactors}

Satuf et al. [21] reported the HG asymmetry factor as a wavelength-dependent magnitude for different commercial brands of titanium dioxide photocatalyst. By combining the solar radiation spectrum taken from [40] with the data reported by Satuf et al., a solar spectral averaged HG asymmetry factor could be obtained by the equation:

$$
g=\frac{\int_{\lambda_{\min }}^{\lambda_{\max }} g_{\lambda} I_{\lambda} d \lambda}{\int_{\lambda_{\min }}^{\lambda_{\max }} I_{\lambda} d \lambda}
$$


where $\lambda_{\min }=295 \mathrm{~nm}$ and $\lambda_{\max }=405 \mathrm{~nm}$ are the upper and lower limits of the interception of the solar radiation and the $\mathrm{TiO}_{2}$ absorption spectrums.

Table 3 reports the average HG asymmetry factor and their corresponding SFM-HG scattering probabilities for three commercial brands of $\mathrm{TiO}_{2}$ studied by Satuf et al. Forward scattering predominates for every photocatalyst. Similar results have been found for $\mathrm{TiO}_{2}$ P25, with $g=0.68$ [30] and $0.3<g<0.9$ depending on wavelengths [17], which suggests that predominantly forward scattering with positives values of $g$ (usually higher than 0.5) are more suitable for describing the scattering phenomenon of irradiated aqueous suspensions of titanium dioxide.

Replacing the solar spectral averaged HG asymmetry factors in Eqs. (27-29) the SFM-HG scattering probabilities were calculated for each commercial photocatalyst (Table 3). In contrast to the SFM-DR [14] with scattering probabilities invariant with catalyst type (Table 3), the SFM-HG can now differentiate between photocatalysts types through the appropriate estimation of the HG asymmetry factor. Therefore, accurate modeling of the radiation field using the SFM-HG is subject to determining, by appropriate experimental methods [21], the HG scattering phase function of the photocatalyst. Therefore, the SFMHG becomes more accurate and precise than simply using the SFM-DR for the modeling the radiation field in photocatalytic reactors. This feature relies on the use of the HG phase function under consideration.

Table 3. SFM-HG scattering probabilities for the average HG asymmetry factor for solar irradiation of aqueous suspension of commercial $\mathrm{TiO}_{2}$ photocatalysts.

$\begin{array}{lllll}\text { Photocatalyst } & g & p_{f} & p_{b} & p_{s}\end{array}$




\begin{tabular}{ccccc}
\hline Aldrich & 0.53 & 0.754 & 0.133 & 0.028 \\
P25 & 0.53 & 0.754 & 0.133 & 0.028 \\
Hombitak & 0.57 & 0.774 & 0.113 & 0.028 \\
X $^{\mathrm{a}}$ & -- & 0.11 & 0.71 & 0.045 \\
\hline
\end{tabular}

${ }^{\mathrm{a}}$ This row was introduced to compare the SFM-HG against the SFM scattering probabilities reported by Brucato et al. [14] for a Diffuse Reflectance phase function in which for any "X" photocatalyst the scattering probabilities are the same.

The tubular and CPC solar photocatalytic reactors described by Colina-Márquez et al. [6] were considered as illustrative examples, to evaluate the performance of the SFM-HG in the modeling of solar photoreactors. The photoreactors of radius $R_{R}=1.65 \mathrm{~cm}$ were operated with $\mathrm{TiO}_{2}$ Degussa P25 with average optical properties: $\sigma=1295.75 \mathrm{~m}^{2} / \mathrm{kg}, \kappa=$ $174.75 \mathrm{~m}^{2} / \mathrm{kg}$ and scattering albedo $\omega=0.88$, which were calculated from the optical properties of the catalyst [21] combined with the solar radiation spectrum [40]. The incident radiation was $I_{0}=30 \mathrm{~W} / \mathrm{m}^{2}$.

Fig. 9 illustrates the LVRPA distribution in the CPC reactor calculated using the SFM with the diffuse reflectance phase function (SFM-DR) and the SFM-HG adapted to this geometry (see [6] for more details). By close inspection better radiation absorption toward the inner regions of the CPC tube is observed (Fig. 9b). This results directly from the nature of the $\mathrm{HG}$ function, which for $\mathrm{TiO}_{2}$ is described by predominantly forward scattering $(g=$ 0.53), favoring the penetration of photons into the reactor volume, and in consequence a reaching a higher degree of photon absorption. In contrast, the predominantly back scattering nature of the DR phase function causes photon to be redirected to their entry points (see Figure SD3 in Supplementary Data for a schematic representation of the rays 
entering the photoreactor window), leading to a higher loss of photons through the reactor walls.

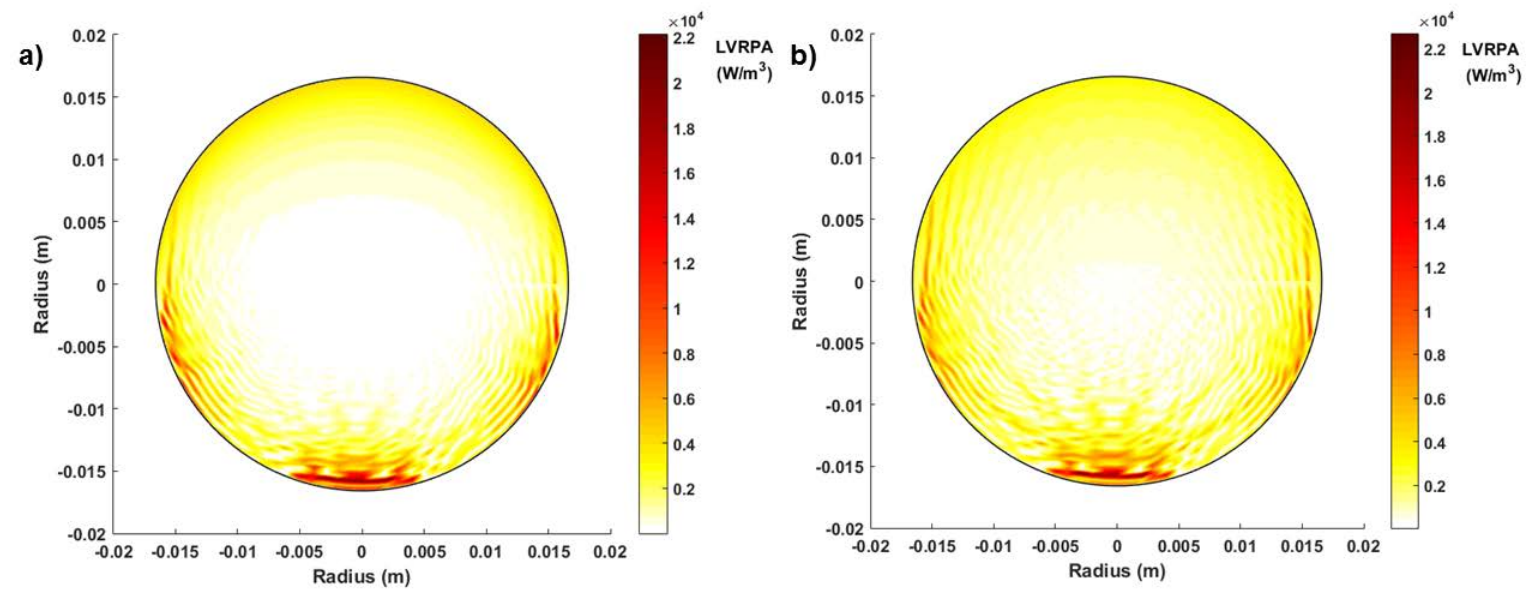

Fig. 9. LVRPA distribution in a CPC photoreactor for $\mathrm{TiO}_{2} \mathrm{P} 25$ as photocatalyst. a) SFM$\operatorname{DR}\left(p_{f}=0.11, p_{b}=0.71, p_{s}=0.045\right)$ b) SFM-HG $g=0.53\left(p_{f}=0.754, p_{b}=0.113, p_{s}=\right.$ 0.028). Catalyst load, $C_{c a t}=0.3 \mathrm{~g} / \mathrm{L}$.

As further verification, the TRPA per unit of reactor length [6], which offers a broader view of the photon absorption in tubulars and CPC photoreactors, was calculated by integration of the LVRPA across the cross section of the CPC reactor:

$$
\text { TRPA / } H=\int_{0}^{2 \pi} \int_{0}^{R_{R}} L V R P A(r, \theta) r d r d \theta
$$

and this yielded $1.12 \mathrm{~W} / \mathrm{m}$ with the SFM-DR model (Fig. 9a) and a higher value equal to 1.45 W/m with the SFM-HG (Fig. 9b) model, since photons penetrate deeper in the inner regions of the reactor. Similar results were determined in the tubular reactor, as shown in Figure SD4 in Supplementary Data. 


\subsubsection{Phase functions and their impact on the optimization of radiation absorption of solar photoreactors}

The impact of different phase functions on the radiation field of photoreactors has recently been investigated in literature [16,17], however, there is a lack of information on its significance with regards to the optimization of radiation absorption is solar photoreactors.

Derived from the SFM approach, the apparent optical thickness $\tau_{\text {app }}$ has been proposed as a key parameter for the design and optimization of photocatalytic reactors [6,35]. $\tau_{\text {app }}$ includes the same advantages related to the use of the optical thickness as a design parameter (i.e. the removal of the effect of the catalyst concentration and reactor length/diameter on the prediction of an optimal radiation field), however in addition, it also removes the effect of the scattering albedo $\omega[6,35]$. In other words, $\tau_{\text {app }}$ incorporates in one single parameter the effect of catalyst loading, reactor dimension and scattering albedo, allowing easy design and optimization of photocatalytic reactors. $\tau_{\text {app }}$ for the tubular and CPC configurations is given in terms of the tube diameter $\left(2 R_{R}\right)$ as $\tau_{\text {app,max }}[6]$ :

$$
\tau_{\text {app, max }}=a \tau_{\text {max }} \sqrt{1-\omega_{\text {corr }}^{2}}
$$

where $\tau_{\max }$ is the maximum optical thickness, defined as:

$$
\tau_{\text {max }}=\beta C_{\text {cat }}\left(2 R_{R}\right)
$$

with $\beta$ the specific extinction coefficient averaged across the solar radiation spectrum and $C_{\text {cat }}$ the catalyst load. Fig. 10 reports the TRPA/H vs $\tau_{\text {app,max }}$ as a function of the scattering phase function and asymmetry factor for CPC photoreactors, operated with $\mathrm{TiO}_{2}$ Degussa P25 (scattering albedo $\omega=0.88$ ). It is observed, that the total radiation absorption increases with forward scattering (greater values of $g$ ), which facilitates the penetration of photons in 
the inner region of the reactor tube. In addition, it is also shown that the value of $\tau_{\text {app,max }}$ which maximizes radiation absorption in the CPC (i.e. the optimum value at which the photoreactor should be operated), is not affected by the phase function or scattering mode. The optimum $\tau_{\text {app,max }}=12$ is significantly higher than that for tubular reactor $\left(\tau_{\text {app,max }}=7\right.$ ) and a flat-plate ( $\tau_{\text {app }}=4.5$ ) since the proven superior radiation absorption for a CPC allows this reactor to be able to bear more catalyst load before reaching the maximum value of radiation absorption. This is attributed, using common language, to the clouding effect due to the increase in the amount of suspended particles [6], a very relevant feature because in heterogeneous photocatalysis reaction rates are expected to be proportional to the catalyst concentration [41]. This outcome agrees with an early study by Li Puma and Brucato [35] who demonstrated through the comparison of the SFM and the Two Flux Model (TFM) (TFM is a singularity of the SFM with $p_{f}=0, p_{b}=1, p_{s}=0$, i.e. photons are totally backscattered,) that the optimum apparent optical thickness for an annular reactor was invariant regardless of the radiation model used, SFM or TFM. This is because the parameters $\omega_{\text {corr }}$ and $a$ in Eq. 34 incorporate the scattering nature through the SFM/SFMHG scattering probabilities (see Eqs. (12-14)).

In summary, the results here presented confirm the apparent optical thickness, $\tau_{\text {app }}$ or $\tau_{\text {app,max }}$ for tubes, as the most suitable parameter for photocatalytic reactor design, operation and optimization, because such parameter is insensitive to scattering albedos and phase functions. This analysis can be easily extended to the tubular configuration and the results are reported in Figure SD5 of Supplementary Data. 


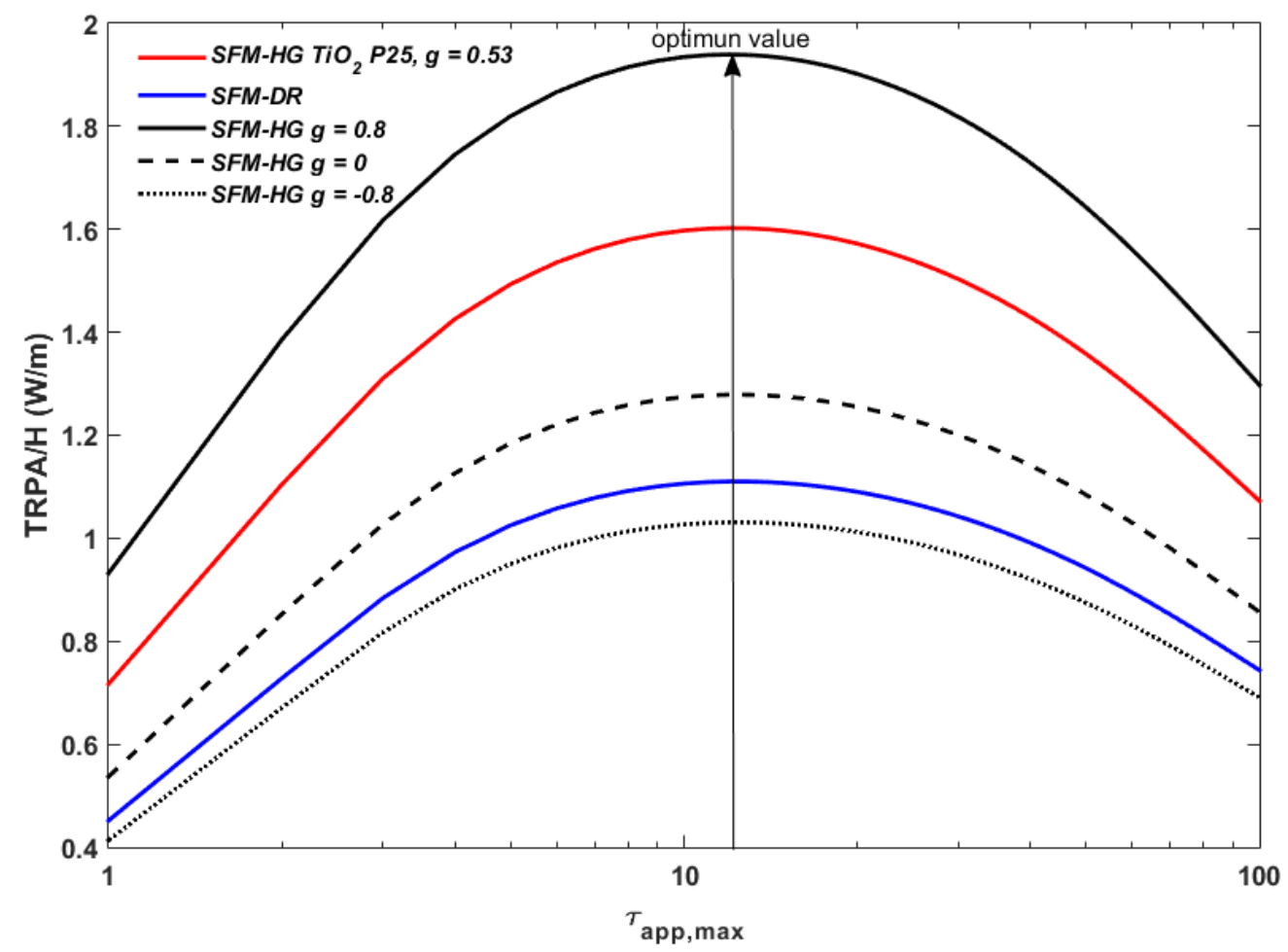

Fig. 10. Effect of the scattering mode/phase function on the optimum maximum apparent optical thickness. The profiles were calculated by keeping constant the optical properties reported for $\mathrm{TiO}_{2} \mathrm{P} 25\left(\sigma=1295.75, \kappa=174.75 \mathrm{~m}^{2} / \mathrm{kg}, \omega=0.88\right)$, while the scattering mode was varied by changing the HG asymmetry factor and including the DR phase function.

\section{Conclusions}

In this study, we have presented the SFM-HG. This new version of the SFM, which incorporates the Henyey-Greenstein phase function, was validated by comparison to Monte Carlo simulations. The SFM-HG retains the simplicity of the SFM approach and adds a more accurate representation of the scattering phenomenon, since it removes the theoretical assumptions of the nature of scattering by the suspended catalytic particles made by the former SFM, relying instead on experimentally determined HG scattering data of 
photocatalysts. The SFM-HG is able to differentiate between photocatalysts, commercial brands and even operational conditions by means of the HG asymmetry factor, which improves the accuracy in radiation field modeling.

In addition, the SFM-HG model allowed the evaluation of the radiation absorption of solar pilot photoreactors and the impact of the scattering phase function. As result, it was shown that forward scattering favors radiation absorption due to better penetration of photons into the reactor volume. The maximum apparent optical thickness derived from the SFMHG/SFM was confirmed as an ideal parameter for photoreactors design and optimization, since it is insensitive to the phase function. It is believed that the SFM-HG represents an advance in the path of simplified modeling and scale-up of photocatalytic systems for environmental, renewable energy and photochemical applications.

\section{Acknowledgements}

The authors thank to Universidad de Cartagena (Colombia), Universidad del Valle (Colombia) and Loughborough University (United Kingdom) by financial support this manuscript. Mueses thanks to COLCIENCIAS of Colombia for support of doctoral studies. Acosta-Herazo thanks to Ch. Engs. Karolina Hurtado and Anabolena Rivera for English revision and artworks.

\section{References}

[1] S. Malato, P. Fernández-Ibáñez, M.I. Maldonado, J. Blanco, W. Gernjak, Decontamination and disinfection of water by solar photocatalysis: Recent overview 
and trends, Catal. Today. 147 (2009) 1-59.

[2] Z. Xing, X. Zong, J. Pan, L. Wang, On the engineering part of solar hydrogen production from water splitting: Photoreactor design, Chem. Eng. Sci. 104 (2013) 125-146.

[3] S. Banerjee, D.D. Dionysiou, S.C. Pillai, Self-cleaning applications of TiO2 by photo-induced hydrophilicity and photocatalysis, Appl. Catal. B Environ. 176-177 (2015) 396-428.

[4] V. Augugliaro, G. Camera-Roda, V. Loddo, G. Palmisano, L. Palmisano, J. Soria, S. Yurdakal, Heterogeneous Photocatalysis and Photoelectrocatalysis: From Unselective Abatement of Noxious Species to Selective Production of High-Value Chemicals, J. Phys. Chem. Lett. 6 (2015) 1968-1981.

[5] A.E. Cassano, O.M. Alfano, Reaction engineering of suspended solid heterogeneous photocatalytic reactors, Catal. Today. 58 (2000) 167-197.

[6] J. Colina-Márquez, F. Machuca-Martínez, G. Li Puma, Radiation absorption and optimization of solar photocatalytic reactors for environmental applications, Environ. Sci. Technol. 44 (2010) 5112-5120.

[7] H.L. Otálvaro-Marín, M.A. Mueses, F. Machuca-Martínez, Boundary layer of photon absorption applied to heterogeneous photocatalytic solar flat plate reactor design, Int. J. Photoenergy. (2014).

[8] R. Siegel, J. Howell, Thermal Radiation Heat Transfer, Third, Hemisphere Publishing Corporation, Washington, DC, 1992. 
[9] Y. Boyjoo, M. Ang, V. Pareek, Some aspects of photocatalytic reactor modeling using computational fluid dynamics, Chem. Eng. Sci. 101 (2013) 764-784.

[10] P.J. Valadés-Pelayo, F. Guayaquil Sosa, B. Serrano, H. de Lasa, Photocatalytic reactor under different external irradiance conditions: Validation of a fully predictive radiation absorption model, Chem. Eng. Sci. 126 (2015) 42-54.

[11] A.L. Loo Zazueta, H. Destaillats, G. Li Puma, Radiation field modeling and optimization of a compact and modular multi-plate photocatalytic reactor (MPPR) for air/water purification by Monte Carlo method, Chem. Eng. J. 217 (2013) 475485.

[12] V. Pareek, S. Chong, M. Tadé, A.A. Adesina, Light intensity distribution in heterogenous photocatalytic reactors, Asia-Pac. J. Chem. Eng. 3 (2008) 171-201.

[13] M.A. Mueses, F. Machuca-Martínez, A. Hernández-Ramirez, G. Li Puma, Effective radiation field model to scattering - Absorption applied in heterogeneous photocatalytic reactors, Chem. Eng. J. 279 (2015) 442-451.

[14] A. Brucato, A.E. Cassano, F. Grisafi, G. Montante, L. Rizzuti, G. Vella, Estimating radiant fields in flat heterogeneous photoreactors by the six flux model, AIChE J. 52 (2006) 3882-3890.

[15] I. Grčic, G. Li Puma, Photocatalytic degradation of water contaminants in multiple photoreactors and evaluation of reaction kinetic constants independent of photon absorption, irradiance, Reactor Geometry and Hydrodinamics, Environ. Sci. Technol. 47 (2013) 13702-13711.

[16] P.J. Valades-Pelayo, J. Moreira, B. Serrano, H. de Lasa, Boundary conditions and 
phase functions in a Photo-CREC Water-II reactor radiation field, Chem. Eng. Sci. 107 (2014) 123-136.

[17] A. Turolla, D. Santoro, J.R. de Bruyn, F. Crapulli, M. Antonelli, Nanoparticle scattering characterization and mechanistic modelling of UV-TiO2 photocatalytic reactors using computational fluid dynamics, Water Res. 88 (2016) 117-126.

[18] M.I. Cabrera, O.M. Alfano, A.E. Cassano, Absorption and Scattering Coefficients of Titanium Dioxide Particulate Suspensions in Water, J. Phys. Chem. 100 (1996) 20043-20050.

[19] R.J. Brandi, O.M. Alfano, A.E. Cassano, Rigorous model and experimental verification of the radiation field in a flat-plate solar collector simulator employed for photocatalytic reactions, Chem. Eng. Sci. 54 (1999) 2817-2827.

[20] R.L. Romero, O.M. Alfano, A.E. Cassano, Radiation field in an annular, slurry photocatalytic reactor. 2. Model and experiments, Ind. Eng. Chem. Res. 42 (2003) 2479-2488.

[21] M.L. Satuf, R.J. Brandi, A.E. Cassano, O.M. Alfano, Experimental method to evaluate the optical properties of aqueous titanium dioxide suspensions, Ind. Eng. Chem. Res. (2005) 6643-6649.

[22] M.H. Yip, M.J. Carvalho, A Monte-Carlo maplet for the study of the optical properties of biological tissues, Comput. Phys. Commun. 177 (2007) 965-975.

[23] G. Li Puma, V. Puddu, H. Tsang, A. Gora, B. Toepfer, Photocatalytic oxidation of multicomponent mixtures of estrogens (estrone (E1), 17 $\beta$-estradiol (E2), 17 $\alpha$ ethynylestradiol (EE2) and estriol (E3)) under UVA and UVC radiation: Photon 
absorption, quantum yields and rate constants independent of photon absorp, Appl. Catal. B Environ. 99 (2010) 388-397.

[24] B. Toepfer, A. Gora, G. Li Puma, Photocatalytic oxidation of multicomponent solutions of herbicides: Reaction kinetics analysis with explicit photon absorption effects, Appl. Catal. B Environ. 68 (2006) 171-180.

[25] J. Marugán, R. Van Grieken, O.M. Alfano, A.E. Cassano, Optical and physicochemical properties of silica supported TiO2 photocatalysts, AIChE J. 52 (2006) 2832-2843.

[26] M.L. Satuf, R.J. Brandi, A.E. Cassano, O.M. Alfano, Scaling-up of slurry reactors for the photocatalytic degradation of 4-chlorophenol, Catal. Today. 129 (2007) 110117.

[27] J. Marugán, R. Van Grieken, A.E. Cassano, O.M. Alfano, Intrinsic kinetic modeling with explicit radiation absorption effects of the photocatalytic oxidation of cyanide with TiO2 and silica-supported TiO2 suspensions, Appl. Catal. B Environ. 85 (2008) 48-60.

[28] J. Marugán, R. van Grieken, C. Pablos, M.L. Satuf, A.E. Cassano, O.M. Alfano, Modeling of a bench-scale photocatalytic reactor for water disinfection from laboratory-scale kinetic data, Chem. Eng. J. 224 (2013) 39-45.

[29] P.J. Valadés-Pelayo, F. Guayaquil Sosa, B. Serrano, H. de Lasa, Eight-lamp externally irradiated bench-scale photocatalytic reactor: Scale-up and performance prediction, Chem. Eng. J. 282 (2015) 142-151.

[30] P.J. Valadés-Pelayo, J. Moreira del Rio, P. Solano-Flores, B. Serrano, H. de Lasa, 
Establishing photon absorption fields in a Photo-CREC Water II Reactor using a CREC-spectroradiometric probe, Chem. Eng. Sci. 116 (2014) 406-417.

[31] G.B. Ortiz de la Plata, O.M. Alfano, A.E. Cassano, Decomposition of 2chlorophenol employing goethite as Fenton catalyst II: Reaction kinetics of the heterogeneous Fenton and photo-Fenton mechanisms, Appl. Catal. B Environ. 95 (2010) 14-25.

[32] G. Li Puma, J.N. Khor, A. Brucato, Modeling of an annular photocatalytic reactor for water purification: oxidation of pesticides, Environ. Sci. Technol. 38 (2004) $3737-3745$.

[33] L. Bressel, O. Reich, Theoretical and experimental study of the diffuse transmission of light through highly concentrated absorbing and scattering materials Part I: Monte-Carlo simulations, J. Quant. Spectrosc. Radiat. Transf. 146 (2014) 190-198.

[34] M. Pasquali, F. Santarelli, J.F. Porter, P.L. Yue, Radiative transfer in photocatalytic systems, AIChE J. 42 (1996) 532-537.

[35] G. Li Puma, A. Brucato, Dimensionless analysis of slurry photocatalytic reactors using two-flux and six-flux radiation absorption-scattering models, Catal. Today. 122 (2007) 78-90.

[36] S.A. Prahl, M. Keijzer, S.L. Jacques, A.J. Welch, A Monte Carlo model of light propagation in tissue, Dosim. Laser Radiat. Med. Biol. 5 (1989) 102-111.

[37] J. Piskozub, D. McKee, Effective scattering phase functions for the multiple scattering regime., Opt. Express. 19 (2011) 4786-94. 
[38] L. Lin, M. Zhang, S. Li, A modified condensed Monte Carlo simulation of reflectance with focus light beam from scattering medium, Int. J. Light Electron Opt. 126 (2015) 4876-4879.

[39] Q. Yang, P.L. Ang, M. Ray, S. Pehkonen, Light distribution field in catalyst suspensions within an annular photoreactor, Chem. Eng. Sci. 60 (2005) 5255-5268.

[40] Standard Tables for Reference Solar Spectral Irradiances: Direct Normal and and Hemispherical on $37^{\circ}$ Tiltled Surface, Astm. 03 (2013) 1-21.

[41] J.M. Herrmann, Fundamentals and misconceptions in photocatalysis, J. Photochem. Photobiol. A Chem. 216 (2010) 85-93. 


\section{Graphical abstract}

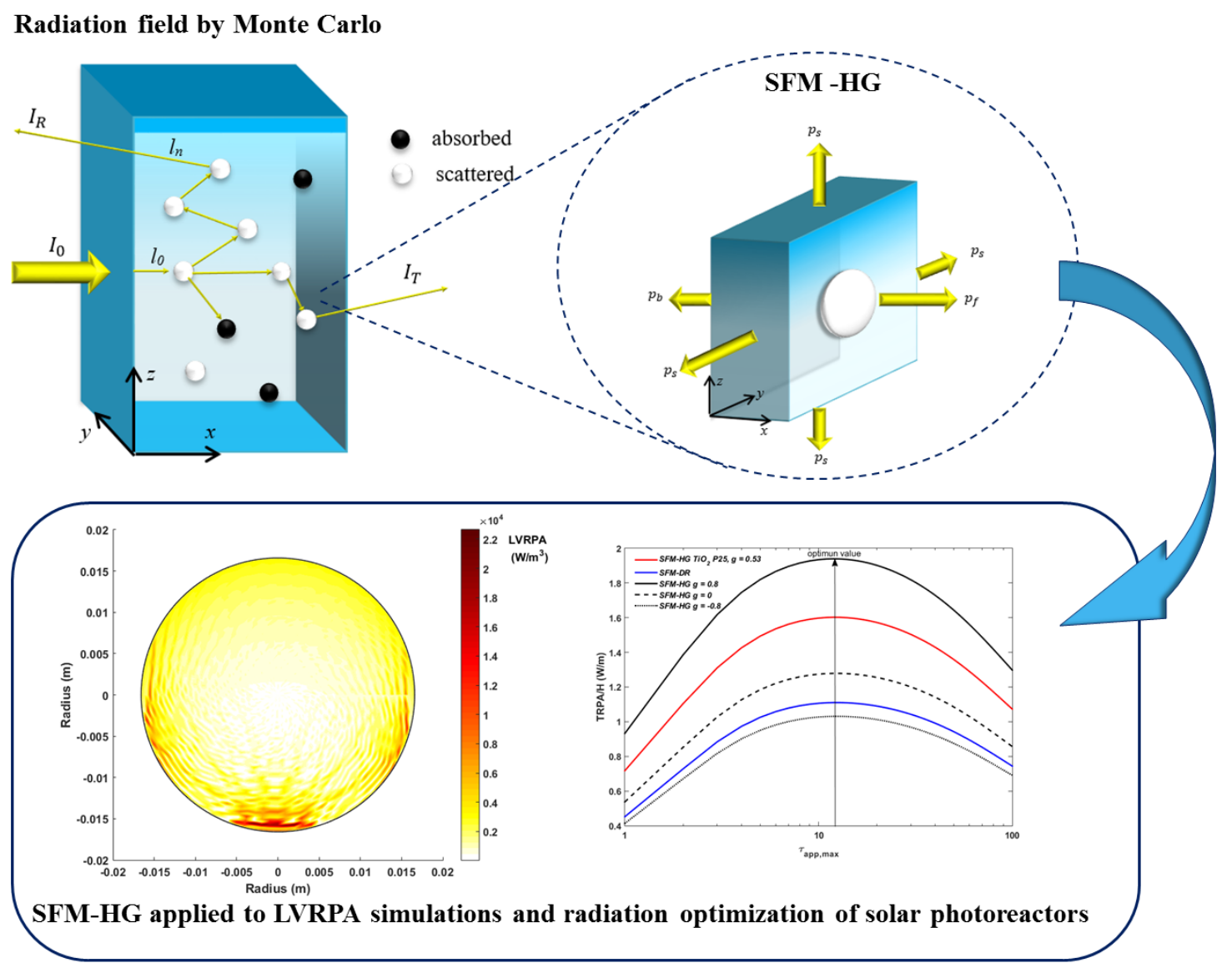




\section{Highlights}

- The coupling of Six Flux Model to the Henyey-Greenstein phase function is achieved.

- The model is validated by comparison to Monte Carlo simulations.

- $\quad$ The model discerns the photocatalysts scattering modes.

- Phase functions evaluated on radiation absorption of solar reactors for first time.

- Photoreactors optimization including the effect of phase functions is presented. 